\title{
TITLE: IDENTITIES FOR GENERALIZED HYPERGEOMETRIC COEFFICIENTS
}

AUTHOR(S): L. C. Biedenharn and J. D. Louck

\section{DISCLAIMER}

This report was prepared as an account of work sponsored by an agency of the United States Government. Neither the United States Government nor any agency thereof, nor any of their employees, makes any warranty, express or implied, or assumes any legal liability or responsibility for the accuracy, completeness, or usefulness of any information, apparatus, product, or process disclosed, or represents that its use would not infringe privately owned rights. Reference herein to any specific commercial product, process, or service by trade name, trademark, manufacturer, or otherwise does not necessarily constitute or imply its endorsement, recommendation, or favoring by the United States Government or any agency thereof. The views and opinions of authors expressed herein do not necessarily state or reflect those of the United States Government or any agency thereof. 
29/September/1991

\title{
Identities for Generalized Hypergeometric Coefficients*
}

\author{
L. C. BIEDENHARN** and J. D. LOUCK \\ Los Alamos National Laboratory, Theoretical Division \\ Los Alamos, N.M., 87545, U.S.A.
}

\begin{abstract}
:
Generalizations of hypergeometric functions to arbitrarily many symmetric variables are discussed, along with their associated hypergenmetric coefficients, and the setting within which these generalizations arose. Identities generalizing the Euler identity for ${ }_{2} F_{1}$, the Saalschütz identity, and two generalizations of the ${ }_{4} F_{3}$ Bailey identity, among others, are given.
\end{abstract}

\section{Introduction}

Hypergeometric functions, denoted by ${ }_{p} F_{q}$, are very well-known in special function theory and the associated 'hypergeometric coefficients' are simply monomial products of gamma functions appearing in the formal power series expansion of ${ }_{p} F_{q}(z)$ over the single variable $z$. The 'generalized hypergeometric coefficients' in our title denotes a generaliza-

* Work performed under the auspices of the U.S. Department of Energy

** Consultant. Duke University, Department of Physics, Durham, N.C., 27706, U.S.A. 
tion to coefficients in a formal power series expansion over arbitrarily many symmetric variables. (The explicit definition is given in Section 2 , below.) In order to emphasize that this generalization is not arbitrary or ad hoc, it is useful to sketch the background and setting within which this generalization arose.

There is at present no overarching general theory of special functions, but several investigations--primarily those of Wigner in the 1950 's - have shown ${ }^{1,2,3}$ that large classes of special functions arise as matrix elements of irreps (ㅍreducible representations) of symmetry groups, such as the quantal rotation group $S U(2)$, so important in theoretical physics. There have been further generalizations of this approach to special functions via symmetry groups (a survey is given in ref. 4), and a particularly fruitful procedure ${ }^{5}$ focusses on tensor operators, rather than irrep matrices, as the fundamental structure. It is this approach which led to the generalization of the hypergeometric functions reported here.

Tensor operators are operators that are most easily defined as acting on a special Hilbert space, called model space (by Gel'fand). Consider the symmetry group of interest for the present work: $S U(3)$, the group of $3 \times 3$ unitary, unimodular matrices. The set of all unitary irreps can be labelled by the set of Young frames $\{\lambda\}$, with $\lambda=\left[\lambda_{1}, \lambda_{2}, 0\right]$, where the $\lambda_{i}$ are nonnegative integers satisfying $\lambda_{1} \geq \lambda_{2} \geq 0$. Let us define $\mathcal{H}_{\lambda}$ as a vector space carrying the irrep $\lambda$. Then model space $\mathcal{H}$ is the direct sum: $\mathcal{H}=\sum_{\lambda \in\{\lambda\}} \oplus \mathcal{H}_{\lambda}$, where each irrep $\lambda$ occurs exactly once in the direct sum. A tensor operator $\mathcal{O}$ is then a bounded map $\mathcal{O}: \mathfrak{h} \rightarrow \mathcal{H}$.

It is a basic result that-by defining an equivariant action on tensor operators by the symmetry group-one may classify tensor operators as sets of operators identified by irrep labels of the group. For tensor operators in $S U(3)$, one accordingly labels a tensor operator by an irrep label $[M]=\left[M_{13}, M_{23}, 0\right] \in\{\lambda\}$, and a triangular pattern of integers: 
$(M) \equiv\left(\begin{array}{c}M_{13} M_{23} 0 \\ M_{12} M_{22} \\ M_{11}\end{array}\right)$, obeying the betweenness constraints $M_{i j} \geq M_{i, j-1} \geq M_{i+1, j}$. (The pattern $(M)$ is called a Gel'fand-Weyl pattern and denotes a unique vector in the irrep $[M]$.) This classification is further refined by a second (inverted triangular) labelling pattern, $(\Gamma) \equiv\left(\begin{array}{c}\Gamma_{11} \\ \Gamma_{12} \Gamma_{22} \\ M_{13} M_{23} 0\end{array}\right)$, whose integer entries also obey the betweenness constraints. (The pattern $(\Gamma)$-called an operator pattern-is structurally the same as $(M)$ but does not have a direct group-theoretic significance.) For unit tensor operators (those having norm one) it has been proved ${ }^{6}$ that: the unit tensor operators in $S U(3)$ are a basis for all $S U(3)$ tensor operators and are canonically labelled (to within \pm signs), by the two patterns $(M)$ and $(\Gamma)$.

A second basic result is that the set of all unit tensor operators (of a given unitary symmetry group) form an algebra, in fact, a non-commutative, but co-commutative, Hopf algebra. This algebra contains as a sub-algebra the universal enveloping algebra of the group. As one might expect, matrix elemerits of these algebras and sub-algebras are a prolific source of special functions. For example, the group $S U(2)$ leads in this way to ${ }_{3} F_{2}$ functions (for the unit tensor operators) and to ${ }_{4} F_{3}$ functions (for invariant products of three coupled unit tensor operators).

The special functions that we discuss below are all associated with tensor operators in $S U(3)$, and arose in defining maps $^{7}$ of canonical unit tensor operators into invariant functions. The most interesting of these invariant functions (related to the denominator function of the canonical form) are a family of rnulti-variable polynomial special functions, denoted by $G_{q}^{t}$ (see section 3 ), which have remarkable symmetry properties among which is a characterization by zeroes that fall into $S U(3)$ weight space patterns ${ }^{7}$. It was the search for a proof of these properties that led to a generalization ${ }^{8}$ of the Gauss hypergeometric function that we now describe.

\section{Symmetric Generalized Hypergeometric Functions}


We begin with a generalization of the Gauss function ${ }_{2} F_{1}(a, b ; c ; z)$. The generalization consists, in essence, of replacing the single variable $z$ in the Gauss series by the Schur functions, $e_{\mu}(z)$, of symmetric function theory ${ }^{9}$, with $z$ denoting a set of $t$ indeterminates $z_{1}, z_{2} \ldots, z_{t}$. The Schur functions are defined in terms of standard Weyl tableaux by the formula:

$$
e_{\mu}(z)=\sum_{\epsilon} z_{1}^{\alpha_{1}} z_{2}^{\alpha_{2}} \ldots z_{t}^{\alpha_{t}}
$$

where $\alpha=\left(\alpha_{1}, \ldots, \alpha_{t}\right)$ is a weight of the partition $\mu$ into $t$ parts. (Equivalently, $\alpha$ is the content of the Young frame of the irrep $[\mu]$ filled in lexically with $1,2, \ldots, t$ according to the usual rules for a standard tableau.) The summation in eq. (2.1) is over all weights $\alpha$ of the $U(t)$ irrep $[\mu]$, including repetitions.

To define our generalized hypergeometric function, denoted ${ }_{2} \mathcal{F}_{1}$, we generalize the Gauss series to be:

$$
{ }_{2} \mathcal{F}_{1}\left(a, b ; c ; z_{1}, z_{2}, \ldots, z_{t}\right) \equiv \sum_{\mu}\left\langle{ }_{2} \mathcal{F}_{1}(a, b ; c) \mid \mu\right\rangle e_{\mu}\left(z_{1}, z_{2}, \ldots, z_{t}\right)
$$

where the generalized hypergeometric coefficients are:

$$
{ }_{2} \mathcal{F}_{1}(a, b ; c)|\mu\rangle \equiv M_{\mu}^{-1} \prod_{s=1}^{t}(a-s+1)_{\mu_{0}}(b-s+1)_{\mu_{0}} /(c-s+1)_{\mu_{\odot}} .
$$

In eq. (2.3), $M_{\mu}$ denotes the measure of the irrep $[\mu]$ defined by

$$
M_{\mu}=(\operatorname{dim} \mu)^{-1} \prod_{i=1}^{t}\left(\mu_{i}+t-i\right) ! /(i-1) !
$$

where $\operatorname{dim} \mu$ denotes the Weyl dimension formula,

$$
\operatorname{dim} \mu=\prod_{i<j}^{t}\left(\mu_{i}-\mu_{j}+j-i\right) / 1 ! 2 ! \ldots(t-1) !
$$

which is also the number of standard tableaux for the Young frame associated to the irrep $[\mu]$. We have also used in eq. (2.3) Pochhammer's notation for the rising factorial, that is, for $a \in \mathbf{N},(x)_{a}=x(x+1) \cdots(x+a-1)=\Gamma(x+a) / \Gamma(x)$ with $(x)_{0}=1$. 
It is easily verified that for $t=1$ definition (2.2) reduces to the classic Gauss series.

The main results proved in Refs. 8 and 10 are the following theorem and two identities.

THEOREM 2.6: The generalized Gauss series obeys the Euler identity:

$$
{ }_{2} \mathcal{F}_{1}(a, b ; c ; z){ }_{2} \mathcal{F}_{1}(c-a-b, d ; d ; z)={ }_{2} \mathcal{F}_{1}(c-a, c-b ; c ; z)
$$

An immediate consequence of this theorem are the following two identities for generalized hypergeometric coefficients:

IDENTITY 2.7: Generalized Saalschütz identity:

$$
\sum_{\mu \nu} g(\mu \nu \lambda)\left\langle{ }_{2} \mathcal{F}_{1}(a, b ; c) \mid \mu\right\rangle\left\langle{ } _ { 2 } \mathcal { F } _ { 1 } \left( c-a-b, d ; d|\nu\rangle=\left\langle{ }_{2} \mathcal{F}_{1}(c-a, c-b ; c) \mid \lambda\right\rangle .\right.\right.
$$

This relation is an easy consequence of the Euler identity and the multiplicative property of the Schur functions,

$$
e_{\mu}(z) e_{\nu}(z)=\sum_{\lambda} g(\mu \nu \lambda) e_{\lambda}(z),
$$

where $g(\mu \nu \lambda)$ denotes the Littlewood-Richardson numbers for $G L(t, \mathbf{C})$.

For $t=1$, we have $g(\mu \nu \lambda)=\delta_{\mu+\nu, \lambda}$, and eq. (2.7) reduces to the classic Saalschütz identity (see Bailey ${ }^{11}$ ).

IDENTITY 2.9: Generalized Bailey Identity of the First Kind:

$$
\begin{gathered}
\sum_{\mu \nu} g(\mu \nu \lambda)\left\langle{ }_{2} \mathcal{F}_{1}(c-a, c-b ; c) \mid \mu\right\rangle\left\langle{ }_{2} \mathcal{F}_{1}\left(c^{\prime}-a^{\prime}, c^{\prime}-b^{\prime} ; c^{\prime}\right) \mid \nu\right\rangle \\
=\sum_{\mu \nu} g(\mu \nu \lambda)\left\langle{ }_{2} \mathcal{F}_{1}(a, b ; c) \mid \mu\right\rangle\left\langle{ }_{2} \mathcal{F}_{1}\left(a^{\prime}, b^{\prime} ; c^{\prime}\right) \mid \nu\right\rangle
\end{gathered}
$$

where the parameters are required to satisfy $c-a-b+c^{\prime}-a^{\prime}-b^{\prime}=0$.

Identity (2.9) is called a generalized Bailey identity because for $t=1$, we have

$$
\begin{aligned}
\sum_{\mu+\nu=\lambda}{ }_{2} \mathcal{F}_{1}(a, b ; c)|\mu\rangle\left\langle{ }_{2} \mathcal{F}_{1}\left(a^{\prime}, b^{\prime} ; c^{\prime}\right) \mid \nu\right\rangle \\
=\frac{(a)_{\lambda}(b)_{\lambda}}{\lambda !(c)_{\lambda}} F_{3}\left(\begin{array}{l}
a^{\prime}, b^{\prime}, 1-c-\lambda,-\lambda ; \\
c^{\prime}, 1-a-\lambda, i-b-\lambda
\end{array}\right) \\
=\frac{\left(a^{\prime}\right)_{\lambda}\left(b^{\prime}\right)_{\lambda}}{\lambda !\left(c^{\prime}\right)_{\lambda}} F_{3}\left(\begin{array}{l}
a, b, 1-c^{\prime}-\lambda,-\lambda ; \\
c, 1-a^{\prime}-\lambda, 1-b^{\prime}-\lambda
\end{array}\right),
\end{aligned}
$$


in which $c-a-b+c^{\prime}-a^{\prime}-b^{\prime}=0$. The identity between the two ${ }_{4} F_{3}$ hypergeometric series (of unit argument) is the reversal identity (reverse the order of terms in the finite series expression). Using (2.10) in identity (2.9) for $t=1$ now gives Bailey's identity ${ }^{11}$. (Shukla ${ }^{12}$ independently obtained relation (2.9).)

It is straightforward to give further generalized hypergeometric functions, corresponding to the standard generalization from ${ }_{2} F_{1}$ to ${ }_{p} F_{q}$. These are the ${ }_{p} \mathcal{F}_{q}$ functions, which we now define.

Let $a=\left(a_{1}, \ldots, a_{p}\right), b=\left(b_{1}, \ldots, b_{q}\right)$, and $z=\left(z_{1}, \ldots, z_{t}\right)$ denote arbitrary complex numerator and denominator parameters, $p$ and $q$ in number, respectively, and $z$ a set of $t$ indeterminates. We define generalized hypergeometric coefficients by

$$
\left\langle{ }_{p} \mathcal{F}_{q}(a ; b) \mid \mu\right\rangle=M_{\mu}^{-1} \prod_{s=1}^{t}\left[\frac{\prod_{i=1}^{p}\left(a_{i}-s+1\right)_{\mu_{e}}}{\prod_{j=1}^{q}\left(b_{j}-s+1\right)_{\mu_{e}}}\right],
$$

where $\mu=\left[\mu_{1} \mu_{2} \ldots \mu_{t}\right]$ is an arbitrary (lexical) partition in $t$ parts and $M_{\mu}$ is the measure factor of eq. (2.4).

These coefficients are now used to define a generalized hypergeometric function by the formal series:

$$
{ }_{p} \mathcal{F}_{q}(a ; b ; z) \equiv \sum_{\mu}\left\langle{ }_{p} \mathcal{F}_{q}(a ; b) \mid \mu\right\rangle e_{\mu}(z),
$$

where $e_{\mu}(z)$ denotes a Schur function in the indeterminates $z_{1}, z_{2}, \ldots, z_{t}$ as defined in eq. (2.1).

One of the simplest special functions that occurs in the ${ }_{p} \mathcal{F}_{q}$ class is ${ }_{1} \mathcal{F}_{0}$. (This function already occurs in the work of Littlewood ${ }^{13}$.) It has the explicit definition given by:

$$
{ }_{1} \mathcal{F}_{0}(a ; z)=\sum_{\mu}\left\langle{ }_{1} \mathcal{F}_{0}(a) \mid \mu\right\rangle e_{\mu}(z)=\prod_{s=1}^{t}\left(1-z_{s}\right)^{a}
$$

where the hypergeometric coefficient is given by

$$
\left\langle{ }_{1} \mathcal{F}_{0}(a) \mid \mu\right\rangle=(\operatorname{dim} \mu) \prod_{s=1}^{t} \frac{(a-s+1)_{\mu_{\rho}}}{(t-s+1)_{\mu_{\rho}}}
$$


It is easily shown that one has:

IDENTITY 2.15: Addition rule:

$$
{ }_{1} \mathcal{F}_{0}(a ; z){ }_{1} \mathcal{F}_{0}(b ; z)={ }_{1} \mathcal{F}_{0}(a+b ; z)
$$

We can now state another identity for generalized hypergeometric coefficients.

IDEnTITy 2.16: Generalized Addition Rule of Binomial Type:

$$
\sum_{\mu \nu} g(\mu \nu \lambda)\left\langle{ }_{1} \mathcal{F}_{0}(x) \mid \mu\right\rangle\left\langle{ }_{1} \mathcal{F}_{0}(y) \mid \nu\right\rangle=\left\langle{ }_{1} \mathcal{F}_{0}(x+y) \mid \lambda\right\rangle
$$

For $t=1$, this relation reduces to

$$
\sum_{\mu+\nu=\lambda} \frac{(x)_{\mu}(y)_{\nu}}{\mu ! \nu^{\prime}}=\frac{(x+y)_{\lambda}}{\lambda !}
$$

hence, the designation of identity (2.16) as a generalized binomial identity.

Before we can state our next identity it is necessary to define the polynomials $G_{q}^{t}$ mentioned in Section 1.

\section{The $G_{q}^{t}$ polynomials of $S U(3)$.}

The invariant polynomials $G_{q}^{t}$ occur in the canonical form ${ }^{7}$ for unit tensor operators in $S U(3)$. Each such unit tensor operator is canonically labelled by the $(M)$ and $(\Gamma)$ patterns (which both contain the irrep labels $\left[\begin{array}{lll}M_{13} & M_{23} & 0\end{array}\right]$ for $S U(3)$, or the labels $\left[\begin{array}{llll}M_{13} & M_{23} & M_{33}\end{array}\right]$ if we generalize to $U(3))$. The operator pattern $(\Gamma)$ specifies that the unit tensor operator in question induces the shifts $\left(\Delta_{1}, \Delta_{2}, \Delta_{3}\right)$ when acting on the irrep $\left[m_{13} m_{23} m_{33}\right]$ of model space; that is $\left[m_{13} m_{23} m_{33}\right] \rightarrow\left[m_{13}+\Delta_{1}, m_{23}+\Delta_{2}, m_{33}+\Delta_{3}\right]$ under the action of the operator. (The relation of the shifts to the $(\Gamma)$ pattern is that $\Delta_{1}=\Gamma_{11}, \Delta_{2}=$ $\Gamma_{12}+\Gamma_{22}-\Gamma_{11}$ and $\Delta_{3}=M_{13}+M_{23}+M_{33}-\Gamma_{12}-\Gamma_{22}$.) The label $t$ in $G_{q}^{t}$ is also determined by the pattern $(\Gamma)$ and canonically labels the multiplicity. 
It is convenient in defining the $G_{q}^{t}$ functions to use a different set of variables than those mentioned above. In place of the irrep variables $\left[m_{13} m_{23} m_{33}\right]$ in model space we first go over to the symmetric variables $\left(p_{i j}+\widehat{j-1}\right)$ (the partial hooks) and then $P_{i j}$ roduce ${ }^{+} j \cdot i$ the differences: $x_{i}=p_{j 3}-p_{k 3}$, where $(i j k)$ is a positive permutation of $\left(\begin{array}{lll}1 & 2 & 3\end{array}\right)$, with $x_{1}+x_{2}+x_{3}=0$. Thus irreps in model space are associated with integer lattice (L) points $\left(x_{1}, x_{2}, x_{3}\right)$ in the Möbius plane $(\mathrm{M})$, and automatically show $S_{3}$ symmetry. The parameters $\left(\Delta_{1}, \Delta_{2}, \Delta_{3}\right)$ now denote shifts in the Möbius plane. Finally the label $M_{13}$ is replaced by $p$ and $M_{23}$ by $q$ (with $M_{33}=0$ ), as a typographic convenience.

Accordingly we have the symbol definitions:

(i) The three-tuple $\left(\Delta_{1}, \Delta_{2}, \Delta_{3}\right)$, such that $\Delta_{i} \in \mathrm{N}, 0 \leq \Delta_{i} \leq p$, and $\Delta_{1}+\Delta_{2}+\Delta_{3}=p+q$, is denoted $\Delta=\left(\Delta_{1}, \Delta_{2}, \Delta_{3}\right)$.

(ii) A point in $\mathbf{L}$ is denoted $x=\left(x_{1}, x_{2}, x_{3}\right)$.

(iii) $\lambda=\left[\lambda_{1} \lambda_{2} \ldots \lambda_{t}\right]$ denotes an irrep label of $U(t)$. The symbols $\mu, \nu, \ldots$ also denote irrep labels with $t$ parts.

(iv) $h(\lambda \mu \nu \rho)$ denotes the number of times irrep $[q-t+1, \ldots, q-t+1]$-this denotes the integer $q-t+1$ repeated $t$ times-is contained in the direct product $\lambda \times \mu \times \nu \times \rho$, and is defined to be zero if $[q-t+1, \ldots, q-t+1] \notin \lambda \times \mu \times \nu \times \rho$. The conjugate irrep $\bar{\lambda}$ has the partition with $\bar{\lambda}_{i}=q-t+1-\lambda_{i}$.

(v) The symbol $A$ denotes the $3 \times 3$ array of variables defined by

$$
A=A_{t}(\Delta ; x)=\left(a_{i j}\right)=\left[\begin{array}{lll}
\Delta_{1}-t+1 & \Delta_{2}-t+1+x_{1} & \Delta_{3}-t+1-x_{1} \\
\Delta_{2}-t+1 & \Delta_{3}-t+1+x_{2} & \Delta_{1}-t+1-x_{2} \\
\Delta_{3}-t+1 & \Delta_{1}-t+1+x_{3} & \Delta_{2}-t+1-x_{3}
\end{array}\right]
$$

(vi) For $t=0$ and $t=q+1$, we define $G_{q}^{0}(\Delta ; x)=G_{q}^{q+1}(\Delta ; x)=1$.

(vii) The notation

$$
\mathcal{G}_{q}^{t}(A)=G_{q}^{t}(\Delta ; x)
$$


is used to signify that $\mathcal{G}_{q}^{t}$ is a polynomial in the variables $a_{i j}$ of the array $A$, hence of $\Delta_{i}$ and $x_{j}$

We can now give explicitly the polynomials $G_{q}^{t}(\Delta ; x), q \in \mathrm{N}, t=1,2, \ldots, q$, using the terms defined above and referring to Section 2 for the definitions of the hypergeometric coefficients.

$$
\begin{aligned}
G_{q}^{t}(\Delta ; x) & =\mathcal{G}_{q}^{t}(A)=\prod_{s=1}^{t} \frac{(q-s+1) !}{(s-1) !} \prod_{i=1}^{3} \prod_{s=1}^{t}\left(-a_{i 1}-s+1\right)_{q-t+1} \\
& \times \sum_{\lambda \mu \nu \rho} h(\lambda \mu \nu \rho)\left\langle{ }_{1} \mathcal{F}_{0}(K-\ell) \mid \rho\right\rangle\left\langle{ }_{2} \mathcal{F}_{1}\left(-a_{12},-a_{13} ; a_{11}-\ell\right) \mid \lambda\right\rangle \\
& \times\left\langle{ }_{2} \mathcal{F}_{1}\left(-a_{22},-a_{23} ; a_{21}-\ell\right) \mid \mu\right\rangle \\
& \times\left\langle{ }_{2} \mathcal{F}_{1}\left(-a_{32},-a_{33} ; a_{31}-\ell\right) \mid \nu\right\rangle
\end{aligned}
$$

where we have defined $\ell=q-2 t+1, K=\Delta_{1}+\Delta_{2}+\Delta_{3}-3 t+3$.

Remark: It is of interest to note that the problem of determining $S U(3)$ tensor operators (and hence the $G_{q}^{t}$ functions) involves generalized hypergeometric functions having arbitrarily large partitions, that is, the Young frames that enter in eq. (3.3) have $t$ rows, corresponding to irreps $[\mu]$ of $U(t)$, with $t$ arbitrarily large (thus not limited to $S U(3)$.)

Let us summarize the properties ${ }^{7}$ of the $G_{q}^{t}$ polynomials:

(i) Total degree $2 t(q-t+1)$ in $x$. By this we mean that $G_{q}^{t}(\Delta ; x)$ is a sum of monomials of the form $x_{1}^{\alpha} x_{2}^{\beta} x_{3}^{\gamma}$, where $\alpha, \beta, \gamma$ are nonnegative integers such that $\alpha+\beta+\gamma \leq$ $2 t(q-t+1)$ and the sum is over all such monomials multiplied by real coefficients that are themselves functions of $\Delta_{i}$. This polynomial property is placed in evidence when eq. (3.3) is rewritten in terms of the quantities (see Ref. 7) $(k \in \mathbf{N})$ :

$$
F_{k, \lambda}(x, y, z)=\prod_{s=1}^{t}(x+t-k-s+1){ }_{k}\left\langle{ }_{2} \mathcal{F}_{1}\left(-y,-z_{;} x+t-k\right) \mid \lambda\right\rangle .
$$

(ii) Determinantal symmetry. 'i'his symmetry refers to the invariance of $G_{q}^{t}(\Delta ; x)$ under the transformation of the six variables $\left(\Delta_{1}, \Delta_{2}, \Delta_{3}, x_{1}, x_{2}, x_{3}\right)$ induced by row interchange, column interchange, and transposition of the $3 \times 3$ array $A$ defined by eq. 
(3.1). For example, under matrix transposition of $A$, that is $A \rightarrow A^{\text {tr }}$, we have

$$
\left(\Delta_{1}, \Delta_{2}, \Delta_{3}, x_{1}, x_{2}, x_{3}\right) \rightarrow\left(\Delta_{1}, \Delta_{2}+x_{1}, \Delta_{3}-x_{1},-x_{1},-x_{3},-x_{2}\right) .
$$

(iii) Weight space $W_{q}^{t}(\Delta)$ of zeroes. The points in $W_{q}^{t}(\Delta)$ are in one-to-one correspondence with those of the weight space of irrep $[q-t, 0,-t+1]$ of $U(3)$. With each point $x \in W_{q}^{t}(\Delta)$, we associate a multiplicity number $M_{q}^{t}(\Delta ; x)$,

$$
M_{q}^{t}(\Delta ; x) \equiv \min \left\{\left(t, q-t+1,1+d_{t}(x)\right\}\right.
$$

where $d_{t}(x)$ is the "distance" from lattice point $x \in W_{q}^{t}(\Delta)$ to the nearest boundary point as measured along the direction of a coordinate axis (one lattice spacing = one unit of distance, with $d_{t}=0$ at the boundary). The multiplicity function $M_{q}^{t}(\Delta ; x)$ assigns to each point $x \in W_{q}^{t}(\Delta)$ exactly the value of the multiplicity of the weight $w=\left(w_{1}, w_{2}, w_{3}\right)$ of irrep $[q-t, 0,-t+1]$, where $w$ is related to the point $x \in W_{q}^{t}(\Delta)$ by $x_{1}=\Delta_{3}-t+1-w_{1}, x_{2}=-\Delta_{2}-\Delta_{3}+q-1-w_{2}, x_{3}=\Delta_{2}-t+1-w_{3}$. By the phrase "a polynomial has the weight space $\|_{q}^{t}(\Delta)$ of zeroes", we mean that each $x \in W_{q}^{t}(\Delta)$ is a zero of the polynomial with multiplicity $M_{q}^{t}(\Delta ; x)$.

Property (i) is already evident from the definition (3.3) of $G_{q}^{t}$, as is the invariance of $G_{q}^{t}(\Delta ; x)$ under the transformation of the variables $(\Delta ; x)$ corresponding to the column (and less obviously row) interchanges in the array $A$. Accordingly, the proof of the determinantal symmetry stated in (ii) requires only the invariance under the transformation (3.5) corresponding to transposition of the array $A$. This transpositional symmetry is also the key to proving that $G_{q}^{t}(\Delta ; x)$ possesses the zeroes described in (iii), a result proved in Ref. 7 .

It was the search for a proof of transpositional symmetry that led to the discovery of many of the special functions discussed in Section 2, although the ${ }_{2} \mathcal{F}_{1}$ generalized hypergeometric function and the associated Saalschütz identity came earlier in developing properties of the $G_{q}^{t}$ polynomials for the special case $t=1$. 
The problem of proving transpositional symmetry can be made more evident if we introduce yet another polynomial function, $A_{\lambda}$. Define new variables $a, b, c, d, e$ by $a=$ $-a_{33}, b=-a_{32}, d=-a_{22}, e=-a_{23}, c=K-\ell$ and define the polynomial $A_{\lambda}$ of these variables by

$$
\begin{aligned}
A_{\lambda} & \left(\begin{array}{l}
a, b, d, e \\
c
\end{array}\right)=\prod_{s=1}^{t}(a+b+c-s+1)_{\lambda_{0}}(d+e+c-s+1)_{\lambda_{d}} \\
& \times \sum_{\mu \nu} g(\mu \nu \lambda)\left\langle{ }_{2} \mathcal{F}_{1}(a, b ; a+b+c) \mid \mu\right\rangle\left\langle{ }_{2} \mathcal{F}_{1}(d+c, e+c ; d+e+c) \mid \nu\right\rangle .
\end{aligned}
$$

We find the following expression for $\mathcal{G}_{q}^{t}$ in terms of the $A_{\lambda}$ functions (using the generalized Saalschütz identity):

$$
\begin{aligned}
\mathcal{G}_{q}^{t}(A) & =(-1)^{t(q-t+1)}\left[\prod_{s=1}^{t} \frac{(q-s+1) !}{(s-1) !}\right] \\
& \times \sum_{\lambda} M_{\lambda}^{-1}\left[\prod_{s=1}^{t}(-1)^{\lambda_{\bullet}}\left(-a_{11}-s+1\right)_{q-t+1-\lambda_{0}}\left(-a_{12}-s+1\right)_{\lambda_{s}}\right. \\
& \left.\times\left(-a_{13}-s+1\right)_{\lambda_{0}}\left(-a_{21}-s+1\right)_{\lambda_{0}}\left(-a_{31}-s+1\right)_{\lambda_{0}}\right] A_{\bar{\lambda}}\left(\begin{array}{l}
a, b, d, e \\
c
\end{array}\right) .
\end{aligned}
$$

The summation in eq. (3.8) is over all partitions $\lambda$ such that $q-t+1 \geq \lambda_{1} \geq \cdots \geq$ $\lambda_{t} \geq 0$. The functions $A_{\lambda}$ given by eq. (3.7) are defined for all partitions, hence for the conjugate partition (see definition (iv) above) that appears in eq. (3.8).

It follows from the discussion above that a sufficient condition for transpositional symmetry of the polynomial $\mathcal{G}_{q}^{t}$, that is, for $\mathcal{G}_{q}^{t}(A)=\mathcal{G}_{q}^{t}\left(A^{\text {tr }}\right)$ is the $b \leftrightarrow e$ symmetry:

$$
A_{\lambda}\left(\begin{array}{l}
a, b, d, e \\
c
\end{array}\right)=A_{\lambda}\left(\begin{array}{l}
a, e, d, b \\
c
\end{array}\right)
$$

which constitutes Identity (4.1) below.

4. Further Identities for Generalized Hypergeometric Functions and Coefficients 
Two basic identities are required to prove the determinantal symmetry of the polynomials $\mathcal{G}_{q}^{t}(A)$ defined and discussed in Section 3. These are the generalized Saalschütz identity (identity (2.7) above) and the generalized Bailey identity of the second kind which we now state.

IDENTITY 4.1: Generalized Bailey Identity of the Second Kind:

$$
\begin{aligned}
A_{\lambda} & \left(\begin{array}{l}
a, b, d, e \\
c
\end{array}\right)=\prod_{s=1}^{t}(a+b+c-s+1)_{\lambda_{0}}(d+e+c-s+1)_{\lambda_{0}} \\
& \times \sum_{\mu \nu} g(\mu \nu \lambda)\left\langle{ }_{2} \mathcal{F}_{1}(a, b ; a+b+c) \mid \mu\right\rangle\left\langle{ }_{2} \mathcal{F}_{1}(d+c, e+c ; d+e+c) \mid \nu\right\rangle \\
& =\prod_{s=1}^{t}(a+e+c-s+1)_{\lambda_{0}}(b+d+c-s+1)_{\lambda_{0}} \\
& \times \sum_{\mu \nu} g(\mu \nu \lambda)\left\langle{ }_{2} \mathcal{F}_{1}(a, e ; a+e+c) \mid \mu\right\rangle\left\langle{ }_{2} \mathcal{F}_{1}(b+c, d+c ; b+d+c) \mid \nu\right\rangle .
\end{aligned}
$$

For $t=1$, this relation reduces to

$$
\begin{aligned}
(c+d)_{n}(c+e)_{n} F_{3}\left(\begin{array}{l}
a, b, 1-c-d-e-n,-n ; \\
a+b+c, 1-c-d-n, 1-c-e-n
\end{array}\right) \\
=(a+c+d)_{n}(a+c+e)_{n} F_{3}\left(\begin{array}{l}
a, a+c, a+b+d+e+2 c+n-1, n ; \\
a+b+c, a+c+d, a+c+e
\end{array}\right),
\end{aligned}
$$

which is again an expression of Bailey's identity ${ }^{11}$. Since identity (4.1) is distinct from identity (2.9), it is called a generalized Bailey identity of the second kind.

Let us remark that the proof of identity (4.1), given in Ref. 14 is equivalent to the proof of the $b, e$ interchange symmetry of the $A_{\lambda}$ coefficients defined by eq. (3.7). This proof we found to be very difficult. The proof was achieved by showing that eq. (3.7) could be expressed in terms of yet another generalization of the hypergeometric functions, very different from the definition in eq. (2.12). In this new form the symmetry in question becomes self-evident. This new generalization (discussed in ref. 5 ) involves a new type of inhomogeneous symmetric function, but space prevents further discussion here.

We conclude this section by noting several summation identities that have been proved for the $A_{\lambda}$ functions: 
SUMMATION IDENTITIES (4.3):

$$
\begin{aligned}
& \text { (a) } \quad A_{\lambda}\left(\begin{array}{l}
0, b, d, e \\
c
\end{array}\right)=\left\langle{ }_{3} \mathcal{F}_{0}(b+c, d+c, e+c) \mid \lambda\right\rangle, \\
& \text { (b) } \quad A_{\lambda}\left(\begin{array}{l}
a, b, 0, e \\
c
\end{array}\right)=\left\langle{ }_{3} \mathcal{F}_{0}(a+c, b+c, e+c) \mid \lambda\right\rangle \text {, } \\
& \text { (c) } \quad A_{\lambda}\left(\begin{array}{l}
a, b, d, e \\
-a
\end{array}\right)=\left\langle{ }_{3} \mathcal{F}_{0}(b, d, e) \mid \lambda\right\rangle \text {, } \\
& \text { (d) } \quad A_{\lambda}\left(\begin{array}{c}
a, b, d, e \\
-d
\end{array}\right)=\left\langle{ }_{3} \mathcal{F}_{0}(a, b, d) \mid \lambda\right\rangle,
\end{aligned}
$$

where

$$
\left\langle{ }_{3} \mathcal{F}_{0}(a, b, c) \mid \lambda\right\rangle=M_{\lambda}^{-1} \prod_{s=1}^{t}(a-s+1)_{\lambda_{0}}(b-s+1)_{\lambda_{0}}(c-s+1)_{\lambda_{0}} .
$$

\section{Concluding Remarks}

Let us conclude by remarking that the results given above may be extended substantially, even without going beyond the group $S U(3)$. We have in mind the generalization which involves $q$-analogs and accordingly the $q$-analog extension of the generalized hypergeometric function ${ }_{p} \mathcal{F}_{q}$; this is quite analogous to the familiar $q$-extension of ${ }_{p} F_{q}$ to $p \phi_{q}$. Recently it has been found there exists a a $q$-generalization of the classical Lie groups to the so-called 'quantum groups' (actually deformations of the universal enveloping algebras). There is a corresponding generalization of tensor operator theory ${ }^{15}$, and from this result it is evident that $q$-analog extensions of all the identities found above must exist as a consequence of the existence of the quantum group $S U_{q}(3)$. Quantum group investigations of such extensions have only just begun, primarily for $q$-analogs of $S U(2)$ results (some of which were obtained by Askey and Wilson ${ }^{16}$ prior to quantum groups); the prospect of $S U(3)$ extensions is both interesting and challenging.

\section{Acknowledgements:}

We would like to thank Professor Donald St.Patrick Richards for his continuing interest in our work and for the invitation to participate in this special session of the A.M.S. 
Tampa Meeting where these results were presented. We would also like to thank Professor

Richards for pointing out the relevance of $/ \mathrm{s}$ work? to the generalization given in eq. (2.2).

\section{References:}

1. E. P. Wigner, Application of Group Theory to the Special Functions of Mathematical Physics, (Unpublished lecture notes, Princeton University, Princeton NJ, 1955).

2. J. Talman, Special Functions: A Group Theoretical Approach, (W. A. Benjamin, NJ, 1968). This monograph is based on ref. 1.

3. N. Vilenkin, Special Functions and the Theory of Group Representations, (Transl. from the Russian, Amer. Math. Soc. Transl. 22, Ameri. Math. Soc., Providence, RI, 1968).

4. L. C. Biedenharn, R. S. Gustafson, M. A. Lohe, J. D. Louck, and S. C. Milne, Special Functions and Group Theory in Theoretical Physics, in Special Functions, Group Theoretical Aspects and Applications, Eds. R. Askey, T. H. Koornwinder, and W. Schempp, (Riedel, NY, 1984) p. 129.

5. J. D. Louck and L. C. Biedenharn, Special Functions Associated with SU(3) WignerClebsch-Gordan Coefficients, Proceedings of the International School on "Symmetry and Structural Properties of Condensed Matter", (September 6-12, 1990, Poznan, Poland), Eds. W. Florek, P. Lulek and M. Nucha, World Scientific (Singapore) 1991.

6. L. C. Biedenharn and J. D. Louck, J. Math. Phys. 13, (1972) 1985.

7. J. D. Louck, M. A. Lohe, and L. C. Biedenharn, J. Math. Phys. 16, (1975) 2408; ibid, J. Math. Phys. 26, (1985) 1458; ibid, 29 (1988) 1106.

8. J. D. Louck and L. C. Biedenharn, J. Math. Anal. 59 (1977) 423.

9. I. G. Macdonald, Symmetric Functions and Hall Polynomials, (Clarendon Press, Oxford, 1979).

10. L. C. Biedenharn and J. D. Louck Adv. in Appl. Math. 9 (1988) 477; ibid, 10 (1989) 396.

11. W. N. Bailey, Generalized Hypergeometric Series, (Cambridge Univ. Press, Cambridge, 1935), p. 56.

12. D. P. Shukla, Indian J. Pure Appl. Math. 12, (1981) 994.

13. D. E. Littlewood, The Theory of Group Characters and Matrix Representations of Groups, (2nd ed., Oxford Univ. Press, London, 1950).

14. L. C. Biedenharn, A. M. Bincer, M. A. Lohe and J. D. Louck (to appear in Adv. in Appl. Math.).

15. L. C. Biedenharn and M. Tarlini, Lett. in Math. Phys. 20 (1990) 271.

16. R. Askey and J. A. Wilson, Some basic hypergeometric orthogonal polynomials that generalize Jacobi polynomials, Memoirs Amer. Math. Soc. 319 (1985). 

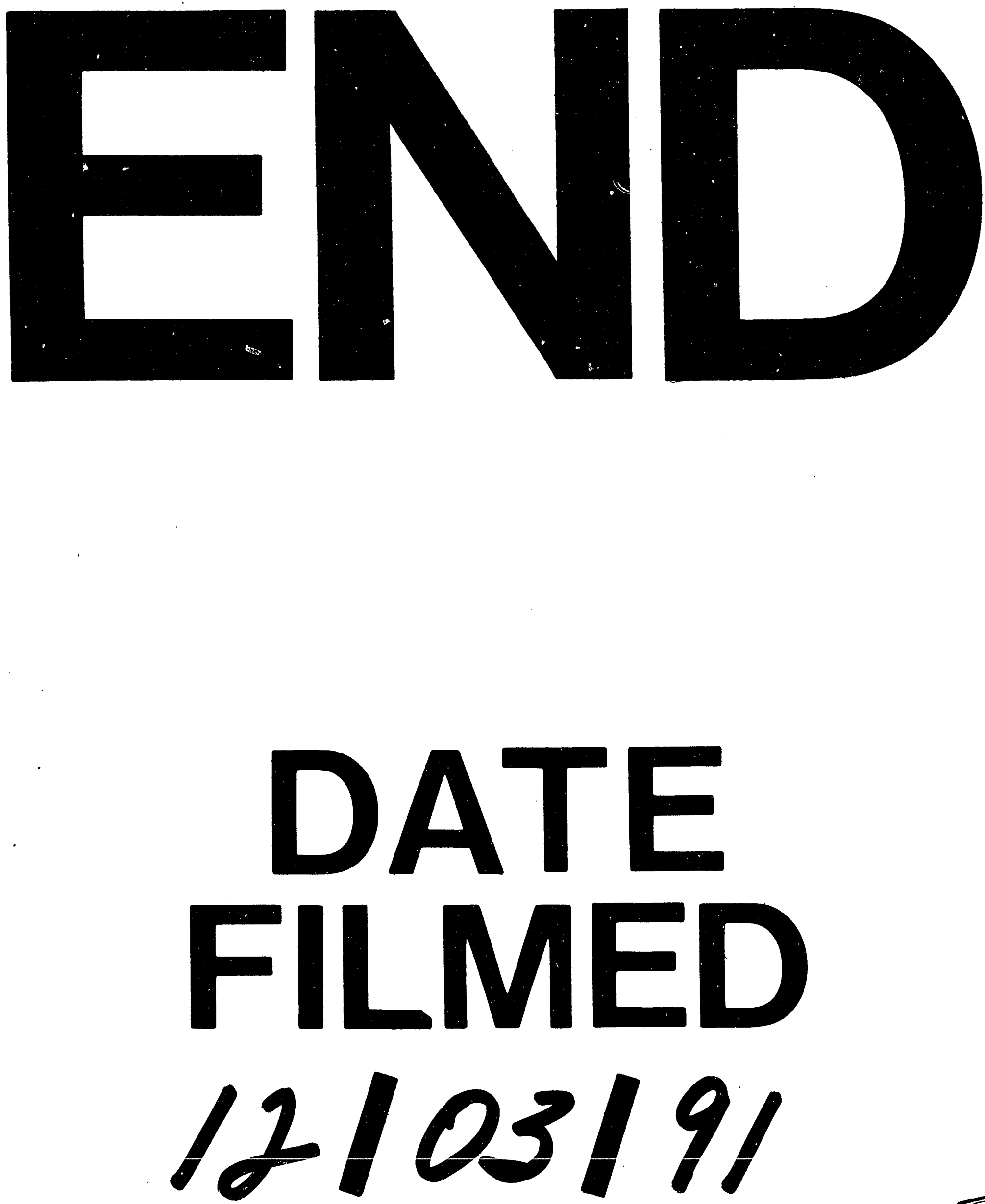
